\title{
Low-energy positron scattering from DNA nucleobases: the effects from permanent dipoles
}

\author{
Jan Franz ${ }^{1, a, b}$ and Francesco Antonio Gianturco $2, \mathrm{c}$ \\ 1 Department of Atomic, Molecular and Optical Physics, Faculty of Applied Physics and Mathematics, Gdansk University \\ of Technology, ul. Narutowicza 11/12, 80-233 Gdansk, Poland \\ 2 Institute of Ion Physics, The University of Innsbruck, Technikerstr. 25, 6020 Innsbruck, Austria
}

Received 23 January 2014 / Received in final form 12 June 2014

Published online 1 October 2014

(C) The Author(s) 2014. This article is published with open access at Springerlink.com

\begin{abstract}
Ab initio quantum calculations for low-energy positron scattering from gas-phase isolated molecular nucleobases which are part of the DNA structure are presented and discussed over the range of $1 \mathrm{eV}$ to $25 \mathrm{eV}$. The calculations report the integral cross sections (ICSs) and the momentum-transfer cross sections (MTCSs) for Adenine, Guanine, Thymine and Cytosine. The calculations show very clearly the important role of the dominant long-range interaction between the positron projectile and the permanent dipole-moments of the target molecules in deciding the relative sizes of the ICSs and MTCSs for the present series of molecules. Such results confirm the largely repulsive interaction between positron and DNA bases, which is nevertheless producing very large cross sections and marked deflection functions from the latter molecules.
\end{abstract}

\section{Introduction}

There has been a remarkable growth in recent years of a great variety of fundamental studies, at the experimental and theoretical/computational level, aimed at investigating the effects that low-energy charged particles may generate when entering the human body and, in general, biological material [1]. These studies are naturally triggered by wanting to follow more in depth the effects of medical therapies or of medical diagnostic tests on live material and on biosystems. While most medical traditional devices initially start with high-energy photons (e.g. with $\mathrm{X}$-rays), or with high-energy electrons or positrons (as in Positron-Emission-Tomography, PET), the impinging radiation quickly thermalises within the body through processes like direct ionization of the molecular environments which leads in turn to the liberation of a substantial number of slower, secondary electrons. Such less energetic particles may therefore attach to various components of the molecular DNA thereby causing important cell and tissue

\footnotetext{
* Contribution to the Topical Issue "Nano-scale Insights into Ion-beam Cancer Therapy", edited by Andrey V. Solov'yov, Nigel Mason, Paulo Limão-Vieira and Malgorzata Smialek-Telega.

${ }^{a}$ Also at: Interdisciplinary Laboratory for Computational Science, FBK-CMM, via Sommarive 18, 38123 Povo, Trento, Italy

b e-mail: j.franz@ucl.ac.uk

c Also at: Scuola Normale Superiore, Piazza dei Cavalieri 7, 56126 Pisa, Italy
}

damages [2]. The similar situation with near-thermal or with low-energy positrons becomes also of fundamental interest since the electron's antiparticle is causing a different variety of processes at the molecular level, annihilation and positronium formation being among them [3]. This means that to have reliable information of the interaction probabilities between such slowed-down positrons and the molecular components of cellular DNA inevitably helps to gain a better understanding of the response at the nanoscopic level of the human material exposed to such specific charged particles since it would allow us to compare such findings with the much larger range of molecualr information which has been gathered for sloweddown electrons in the last ten years or so [1]. In the present work we therefore intend to present a theoretical and computational analysis of the effects of the interactions between low-energy positrons, i.e. up to about $25 \mathrm{eV}$ of initial energy, and the DNA nucleotides that have been already extensively studied by electron scattering around the same range of collision energies. The hope is that, based on what we have been able to find out already in similar molecular systems for which experimental data exist for comparison and for which our computational approach has been proved to be quite reliable in yielding good agreement with such data, the future acquisition of new experimentally measured integral cross sections for low-energy positron scattering could be guided by the computational findings of the present work. We shall show, in fact, that the dominant interaction component of the forces at play between the slow positrons and the nucleobases is provided by the 
permanent dipole moments of such molecules and by the very long-range nature of such interaction that decays as about $r^{-2}$ and has the simple angular dependence of the $n=1$ Legendre Polynomial. Hence, one expects to provide a simple scaling law of the relative sizes of the relevant integral cross sections summed over rotational excitation processes in the gas phase, the latter being a sort of zeroth order approximation to the actual biological environment, as often discussed in the relevant literature [1].

Under experimental conditions one will always find a mixture of various tautomers and conformers. Since all interactions between the positron and the molecule are depending on the molecular structure, the cross sections will be different for different geometries of the target molecule.

We have studied one tautomer of each DNA nucleobase by means of a model potential approach, in which all electro-static, correlation and polarization interactions between the positron and the molecular electrons and nuclei is taken into account. It turns out, that in the energy range between 1 and $25 \mathrm{eV}$ the cross sections are structureless. The interaction between the positron and the target molecule is dominated by the long-range dipole potential and the cross sections can be approximated by the first-order Born approximation. Therefore we are using the Born approximation to compute the cross sections for various tautomers.

The work is organized as follows: Section 2 describes briefly our theoretical framework and its computational implementation, while the Section 3 presents and discusses our results for all the nucleobases of importance in DNA studies. Our present conclusions are summarized in Section 4.

\section{Theoretical and computational methods}

\subsection{Scattering equations}

The total wavefunction $\Psi$ at a given energy $E$ can be obtained by solving the Schrödinger equation

$$
H \Psi=E \Psi .
$$

The total Hamiltonian is given by

$$
H=H_{\mathrm{mol}}+K+V \text {, }
$$

where the operators for the molecular Hamiltonian, the kinetic energy of the colliding positron and the interaction between the positron and the molecule are given by $H_{\mathrm{mol}}$, $K$ and $V$, respectively. In this study we are excluding molecular vibrations, electronic excitations and ionization. Therefore the molecular Hamiltonian is equal to the rotational Hamiltonian

$$
H_{\text {mol }}=H_{\text {rot }} .
$$

In the body-fixed (BF) frame the total wavefunction can be written as

$$
\Psi\left(\mathbf{r}_{1} \ldots \mathbf{r}_{Z}, \mathbf{r}_{p} \mid \mathbf{R}\right)=\Psi_{\mathrm{mol}}\left(\mathbf{r}_{1} \ldots \mathbf{r}_{Z} \mid \mathbf{R}\right) \varphi\left(\mathbf{r}_{p} \mid \mathbf{R}\right) .
$$

Here $\mathbf{r}_{1} \ldots \mathbf{r}_{Z}$ and $\mathbf{r}_{p}$ are the position vectors of the $Z$ bound electrons and the positron, respectively. $\Psi_{\mathrm{mol}}\left(\mathbf{r}_{1} \ldots \mathbf{r}_{Z} \mid \mathbf{R}\right)$ refers to the electronic wavefunction of the target at the nuclear geometry $\mathbf{R}$. The continuum wavefunction for the positron is expanded around a single center (SCE)

$$
\varphi\left(\mathbf{r}_{p} \mid \mathbf{R}\right)=\sum_{l \pi \mu h} r_{p}^{-1} u_{l h}^{\pi \mu}\left(r_{p} \mid \mathbf{R}\right) X_{h l}^{\pi \mu}\left(\hat{\mathbf{r}}_{p}\right) .
$$

The $X_{h l}^{\pi \mu}$ are symmetry-adapted angular basis functions for the component $\mu$ of the irreducible representation $\pi$ with angular momentum quantum number $l$ and angular momentum component $h$ (for more details see e.g. Gianturco and Jain [4]). The functions $u_{l h}^{\pi \mu}$ are the corresponding radial parts of the wavefunction.

In this study we consider collision energies above $1 \mathrm{eV}$. In this energy range molecular rotations are slow when compared with the velocity of the colliding positron. Therefore the geometry of the target molecule can be kept fixed during the collision. This is called the fixednuclei (FN) approximation and ignores the term $H_{m o l}$ in equation (2). To solve the Schrödinger equation in the FN approximation the body-fixed $(\mathrm{BF})$ frame of reference is used instead of he space-fixed (SF) frame, because the equations are simpler and can be solved computationally more efficiently. The two frames are related to each other through a frame transformation, as given for example by Chang and Fano [5].

In the following the index $v$ represents $(\pi \mu h)$ collectively. Substituting equations (4) and (5) into (1) and using the FN approximation, a set of coupled differential equations for the radial functions is obtained

$$
\begin{aligned}
\left\{\frac{d^{2}}{d r_{p}^{2}}-\frac{l(l+1)}{r_{p}^{2}}+k^{2}\right\} & u_{l v}\left(r_{p} \mid \mathbf{R}\right) \\
& =2 \sum_{l^{\prime} v^{\prime}}\left\langle l v|\mathbf{V}| l^{\prime} v^{\prime}\right\rangle u_{l^{\prime} v^{\prime}}\left(r_{p} \mid \mathbf{R}\right)
\end{aligned}
$$

Here the coupling potential is given by

$$
\left\langle l v|\mathbf{V}| l^{\prime} v^{\prime}\right\rangle=\int d \hat{r}_{p} X_{l v}^{*}\left(\hat{r}_{p}\right) V\left(r_{p} \mid \mathbf{R}\right) X_{l^{\prime} v^{\prime}}\left(\hat{r}_{p}\right)
$$

Solving equation (6) with a close-coupling approach under the boundary conditions that the asymptotic form of the radial functions $u_{l v}$ is a sum containing outgoing spherical Bessel- and Neumann-functions, the $K$-matrix elements $K_{l^{\prime} v^{\prime}}^{v v}$ are obtained. The numerical algorithm is described in more detail in references $[6,7]$. The integral cross section (ICS) for the elastic scattering in the BF frame is given by

$$
\sigma_{\mathrm{cc}}=\frac{\pi}{k^{2}} \sum_{l v} \sum_{l^{\prime} v^{\prime}}\left|T_{l^{\prime} v^{\prime}}^{l v}\right|^{2},
$$

where the index cc indicates the close-coupling approach. The $T$-matrix is defined as a function of the $K$-matrix as,

$$
\mathbf{T}=1-(1-i \mathbf{K}) \cdot(1+i \mathbf{K})^{-1}
$$


The integral cross section diverges in the forward scattering direction in the presence of a molecular dipole moment, because of the long-range interaction between the positron and the molecular dipole moment. This problem can be solved by applying the following closure formula for the differential cross section [8]:

$$
\begin{aligned}
\frac{d \sigma}{d \Omega}\left(J \tau \rightarrow J^{\prime} \tau^{\prime}\right)= & \frac{d \sigma_{\mathrm{rd}}^{B}}{d \Omega}\left(J \tau \rightarrow J^{\prime} \tau^{\prime}\right) \\
& +\sum_{L}\left(A_{L}-A_{L}^{B}\right) P_{L}(\cos \theta),
\end{aligned}
$$

where $J \tau$ and $J^{\prime} \tau^{\prime}$ denote the initial and final rotational level, respectively. The first quantity on the right hand side is the differential cross section for a rotating dipole computed with the first Born approximation. The $P_{L}(\cos \theta)$ are the Legendre functions. The coefficients $A_{L}$ are computed from the $K$-matrices, which are obtained by solving the close-coupling equations. The coefficients $A_{L}^{B}$ are computed from the $K$-matrices using the first Born approximation. Explicit formulas for $A_{L}$ and $A_{L}^{B}$ are given in Gianturco and Jain [4]. In the publications by Gianturco and Jain [4] and by Sanna and Gianturco [8] it is assumed that the molecular dipole moment is aligned along one of the main axis of inertia. This assumption is in general not valid for molecules with low molecular symmetry, e.g. having point group symmetries $C_{\mathrm{s}}$ or $C_{1}$. Therefore the expressions for the Born-corrected $K$-matrix elements, the differential, integral and momentum tranfer cross sections have to be modified. We give explicit formulas in the appendix. All formulas have been implemented into a local version of the program POLYDCS, which is used here to perform the frame transformations and to compute the rotational elastic and inelastic cross sections.

The final differential cross section is obtained by summation over the initial and final rotational levels. In this study all molecules are initially in the lowest rotational state $(J=0, \tau=0)$, because the effects on the cross section of populating higher rotationally excited states are less than a few percent for temperatures up to $500 \mathrm{~K}$, as we have shown earlier for positron scattering from pyrimidine [9].

$$
\frac{d \sigma}{d \Omega}=\sum_{J^{\prime} \tau^{\prime}} \frac{d \sigma}{d \Omega}\left(00 \rightarrow J^{\prime} \tau^{\prime}\right)
$$

The corresponding integral cross section in the SF frame can be computed as

$$
\begin{aligned}
\sigma & =\sigma_{\mathrm{rd}}^{B}+\sigma_{\mathrm{cc}}-\sigma_{\mathrm{fd}}^{B} \\
& =\sigma^{B}+4 \pi\left(A_{0}-A_{0}^{B}\right) .
\end{aligned}
$$

Here $\sigma_{r d}^{B}$ is the integral cross section for a rotating dipole in the Born approximation. $\sigma_{c c}$ is the integral cross section obtained by solving the close-coupling equations in the FN-approximation and $\sigma_{f d}^{B}$ is the integral cross section for a fixed dipole. Further details can be found in Sanna and Gianturco [8].

\subsection{The modelling of correlation and polarization by density functional theory}

The interaction between the positron and the molecular nuclei and electrons is given by the total interaction potential

$$
V_{\text {tot }}\left(\mathbf{r}_{\mathrm{p}} \mid \mathbf{R}\right)=V_{\mathrm{st}}\left(\mathbf{r}_{\mathrm{p}} \mid \mathbf{R}\right)+V_{\mathrm{pcp}}\left(\mathbf{r}_{\mathrm{p}} \mid \mathbf{R}\right),
$$

which is the sum of the static potential $V_{\text {st }}$ and the correlation-polarization potential $V_{\mathrm{pcp}}$. The static potential $V_{\text {st }}$ is the exact electro-static interaction potential between the positron and the nuclei and electrons in the molecule. The correlation-polarization potential is modeled by the potential [7]

$$
V_{\mathrm{pcp}}\left(\mathbf{r}_{\mathrm{e}} \mid \mathbf{R}\right)=\left\{\begin{array}{c}
V_{\text {corr }}\left(\mathbf{r}_{\mathrm{p}} \mid \mathbf{R}\right) \text { for } r_{p} \leq r_{c} \\
V_{\mathrm{pol}}\left(\mathbf{r}_{\mathrm{p}} \mid \mathbf{R}\right) \text { for } r_{p}>r_{c}
\end{array} .\right.
$$

Here $V_{\text {corr }}$ and $V_{\text {pol }}$ are the short-range part and longrange parts of the correlation-polarization potential. $r_{c}$ is the outermost point, at which $V_{\text {pol }}$ becomes larger than $V_{\text {corr }} . V_{\text {corr }}$ is based on the functional $\varepsilon^{\mathrm{e}-\mathrm{p}}\left[\rho\left(\mathbf{r}_{\mathrm{p}} \mid \mathbf{R}\right)\right]$ for the correlation energy of one positron in an electron gas with density $\rho\left(\mathbf{r}_{\mathrm{p}} \mid \mathbf{R}\right)$. Boronski and Nieminen [10] have derived interpolation formulae for $\varepsilon^{\mathrm{e}-\mathrm{p}}$. $V_{\text {corr }}$ can be obtained from $\varepsilon^{\mathrm{e}-\mathrm{p}}$ by the functional derivative [7]

$$
V_{\text {corr }}\left(\mathbf{r}_{\mathrm{p}} \mid \mathbf{R}\right)=\frac{\delta}{\delta \rho}\left\{\varepsilon^{\mathrm{e}-\mathrm{p}}\left[\rho\left(\mathbf{r}_{\mathrm{p}} \mid \mathbf{R}\right)\right]\right\} .
$$

More informations can also be found in e.g. Franz et al. [11]. The long-range part $V_{\text {pol }}$ of the correlationpolarization potential is given by

$$
V_{\mathrm{pol}}\left(\mathbf{r}_{\mathrm{p}} \mid \mathbf{R}\right)=-\frac{1}{2 r_{p}^{6}} \sum_{i j} x_{i} x_{j} \alpha_{i j}
$$

where $\alpha_{i j}$ are the Cartesian elements of the polarizability tensor, $r_{p}=\left|\mathbf{r}_{\mathrm{p}}\right|=\sqrt{\sum_{i} x_{i}^{2}}$ and $x_{i}, x_{j} \in\{x, y, z\}$ are the Cartesian coordinates of the positron.

\subsection{Computational details}

For each nucleabase we have studied one tautomer with the model potential approach. In these studies the target molecules are constrained to equilibrium structures that belong to the point group $C_{\mathrm{s}}$. The molecular geometries are given in Figures 1 and 2. The geometries are optimized with the program package Gaussian 09 using the Perdew-Burke-Ernzerhof (PBE) density functional $[12,13]$ and the basis sets cc-pVTZ or aug-cc-pVTZ [14]. We are using the PBE functional, because of its simplicity, physical motivation [12] and its reliability in obtaining ground state energies and dipole moments which are comparable in accuracy with those obtained with the coupled cluster single doubles (CCSD) method [15]. The molecular orbitals are generated at the same level of theory. All molecular parameters are collected in Table 1 . We have selected 


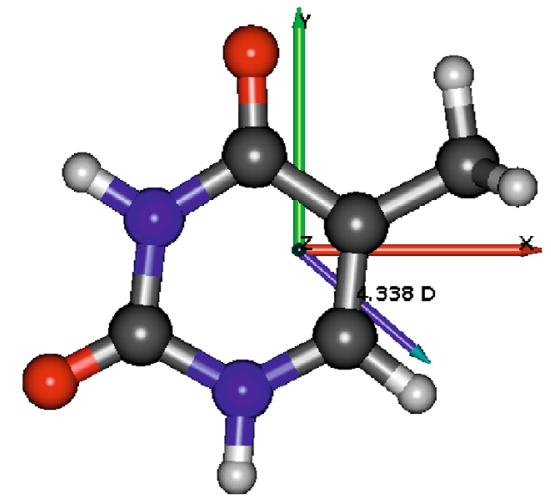

(a) Thymine

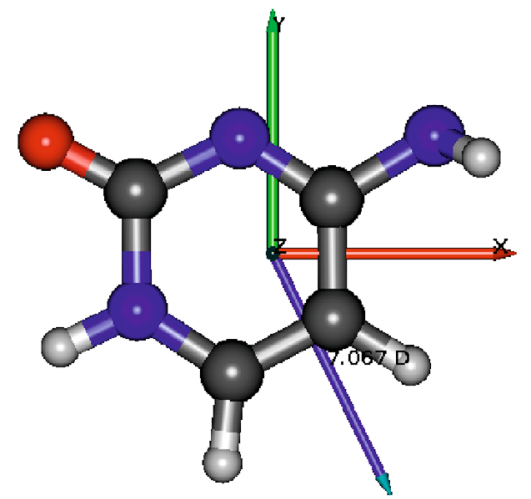

(b) Cytosine

Fig. 1. Molecular geometries of thymine and cytosine. The blue arrow shows the molecular dipole moment in the $x y$ plane.

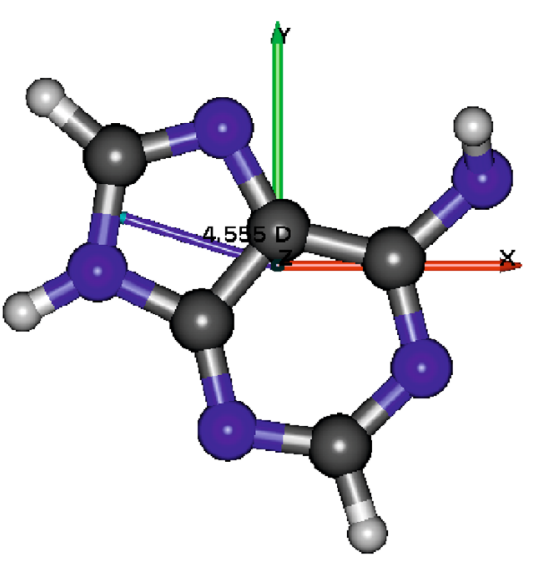

(a) Adenine

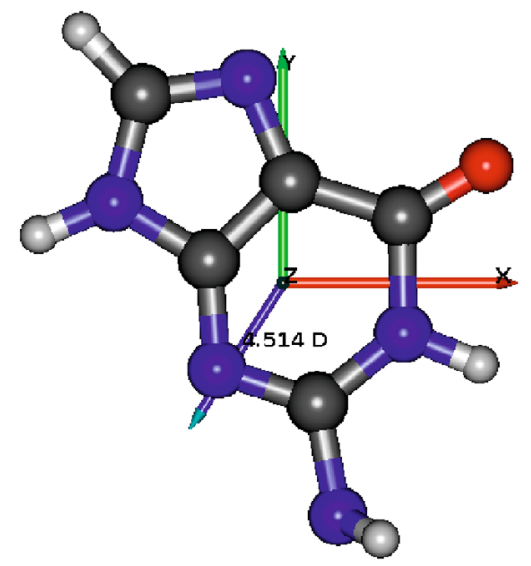

(b) Guanine

Fig. 2. Molecular geometries of adenine and guanine. The blue arrow shows the molecular dipole moment in the $x y$ plane.

Table 1. Molecular parameters. All quantities are computed with the Perdew-Burke-Ernzerhof (PBE) density functional and either with the cc-pVTZ (VTZ) or aug-cc-pVTZ (AVTZ) basis set. Dipole moments are given in Debye, polarizabilities in bohr ${ }^{3}$. The rotational constants $A, B$ and $C$ are given in $\mathrm{GHz}$ and refer to the rotation along the $x, y$ and $z$ axis, respectively. The lowets rotational energy levels $\varepsilon_{J \tau}$ are given in $10^{-6} \mathrm{eV} \cdot \tau=-1,0,1$ are the labels for the sublevels of $J=1$ and refer to rotations around the $x, y$ and $z$ axis, respectively. The ring system of all molecules lies within the $x y$ plane. Due to symmetry $\mu_{z}=0$ and $\alpha_{x z}=\alpha_{y z}=0 . \Delta E$ is the relative energy (given in $\mathrm{kJ} / \mathrm{mol}$ ) with respect to the most stable tautomer given in Tables 2-4.

\begin{tabular}{cccccc}
\hline Molecule & Thymine & Cytosine & \multicolumn{2}{c}{ Adenine } & Guanine \\
\hline Level of theory & PBE/AVTZ & PBE/AVTZ & PBE/AVTZ & PBE/VTZ & PBE/VTZ \\
\hline$\mu_{x}$ & 3.28 & 3.11 & -4.35 & -4.29 & -2.43 \\
$\mu_{y}$ & -2.84 & -6.35 & 1.35 & 1.37 & -3.81 \\
$|\mu|$ & 4.34 & 7.07 & 4.55 & 4.50 & 4.51 \\
$\alpha_{x x}$ & 104.9 & 104.5 & 124.3 & 114.3 & 118.6 \\
$\alpha_{y x}$ & 12.6 & -2.6 & -9.1 & -9.4 & -16.4 \\
$\alpha_{y y}$ & 105.6 & 86.6 & 114.9 & 106.4 & 122.8 \\
$\alpha_{z z}$ & 51.8 & 47.8 & 56.0 & 48.8 & 50.8 \\
$A$ & 3.162 & 3.844 & 2.307 & 2.308 & 1.903 \\
$B$ & 1.389 & 1.957 & 1.563 & 1.566 & 1.097 \\
$C$ & 0.971 & 1.306 & 0.936 & 0.937 & 0.698 \\
$\varepsilon_{1-1}$ & 18.823 & 23.989 & 16.008 & 16.022 & 12.406 \\
$\varepsilon_{10}$ & 17.095 & 21.296 & 13.415 & 13.424 & 10.759 \\
$\varepsilon_{11}$ & 9.759 & 13.493 & 10.338 & 10.352 & 7.423 \\
$\Delta E$ & 0.0 & 22.865 & - & 38.171 & 17.346 \\
\hline
\end{tabular}




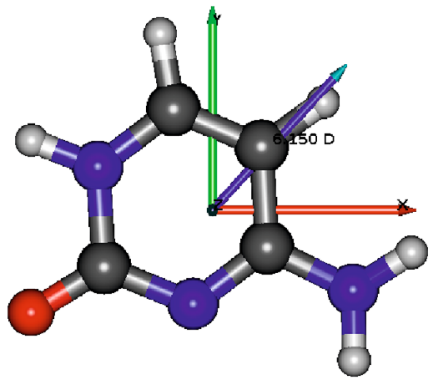

(a) 1

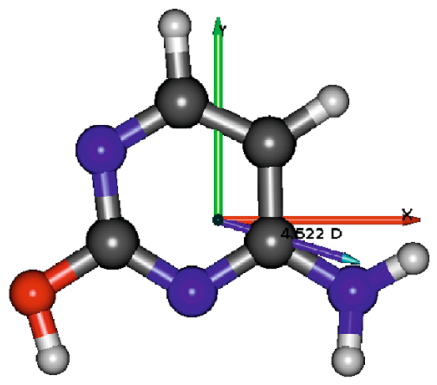

(b) $2 \mathrm{a}$

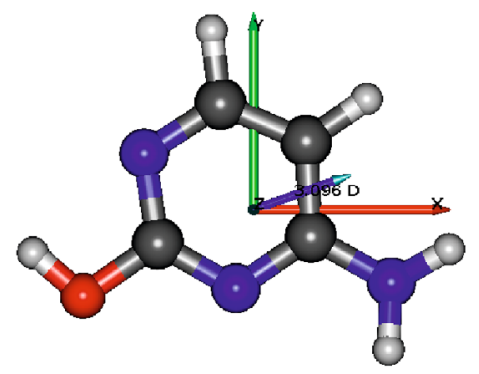

(c) $2 \mathrm{~b}$

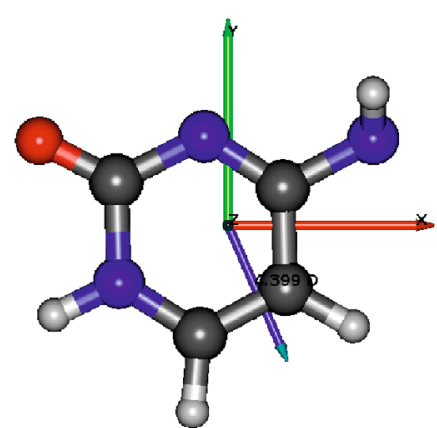

(d) $3 \mathrm{a}$

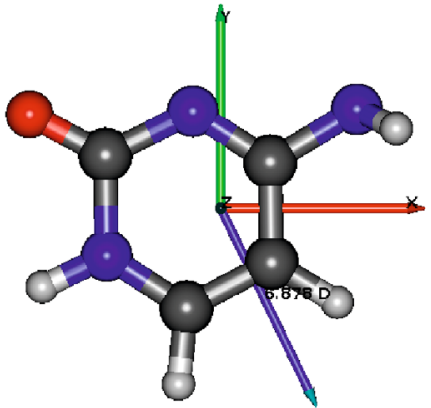

(e) $3 \mathrm{~b}$

Fig. 3. Molecular geometries of various tautomers of cytosine. The blue arrow shows the molecular dipole moment in the $x y$ plane.

these tautomers for a more detailed study of the numerical convergence and to check the validity of the Born approximation, because the geometry has a higher symmetry (point group $C_{\mathrm{s}}$ ) than the more stable tautomers (point group $C_{1}$ ) disucssed below, and therefore allows for a more efficient compuatation with a larger partial wave expansion.

The single-centre-expansions (SCE) of the molecular electron density and of the potential are done with an improved version of the SCELib3.0 computational library [16], to which we have added the correlationpolarization potential specific for modeling the interactions of the molecular electrons with slow positrons. The coupled scattering equations are solved by Volterra integration, using an improved version of the VOLSCAT program package [17]. The grid for the radial integration ranges up to 521 bohr. More specifically the VOLSCAT suite of codes computes the integral cross section in the $\mathrm{BF}$ frame (denoted previously as $\sigma_{c c}$ ) and therefore generates the necessary $K$-matrices in the BF frame.

The transformation of the $K$-matrices from the BFframe into quantities in the SF frame is done with the program POLYDCS [8]. POLYDCS also applies the Born correction, as outlined in Sanna and Gianturco [8] and using the equations given in the appendix. From the $K$-matrices in the SF frame the state-to-state rotationally elastic and inelastic differential and integral cross sections are computed. During the frame transformation step of the present calculations the rotational eigenfunctions and eigenvalues for the asymmetric top are in turn generated using the program ASYMTOP of Jain and Thompson [18] with our computed rotational constants given in Table 1.
For the study of the various tautomers the same quantum chemistry methods was used, as mentioned above. The geometries of the most stable tautomers of cytosine, guanine and adenine are shown in Figures 3-5. The molecular parameters are given in Tables 2-4. For the computation of the rotational energy levels, the program ASYMTOP is used. The cross sections are computed with the equations given in the appendix. Thymine has only one important tautomer, which is the one shown in Figure 1.

\section{Results and discussion}

The summary of the structural properties of the DNA nucleobases discussed in the present work are reported in Table 1, where the permanent dipole moments for the four molecules are clearly telling us that all of them possess values of it which are much larger than the critical value of about 1.67 Debye and that the cytosine target has the largest dipole value of more than 7 Debyes. This is an interesting set of data when one is reminded that positron scattering at low and very-low collision energies (e.g. around $1 \mathrm{eV}$ and below), is largely controlled by the long-range interactions since the impinging particle feels the repulsive effects of the nuclear charges of the component atoms at the equlibrium geometry of the bound structures while also experiencing the weaker attractive interactions originating from electron-positron Coulomb effects and the dynamical correlation between the bound particles and the interacting antiparticle, as outlined in the previous section, together with the attractive contributions from the dipole polarizabilities of each molecular targets. The latter properties are also shown by the table 


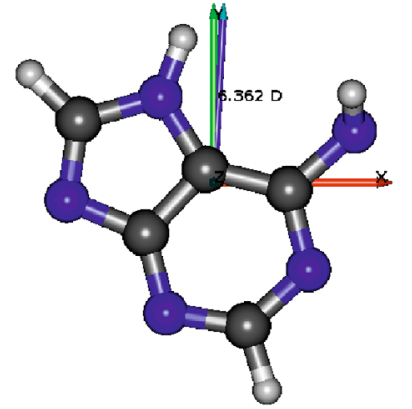

(a) $1 \mathrm{a}$

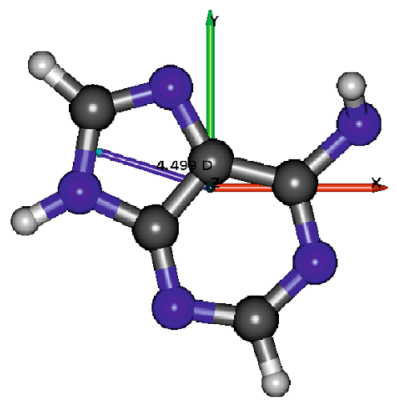

(d) $2 \mathrm{a}$

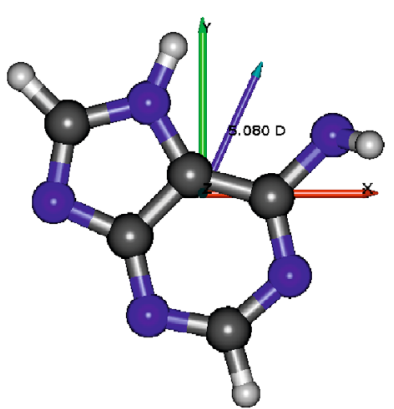

(b) $1 \mathrm{~b}$

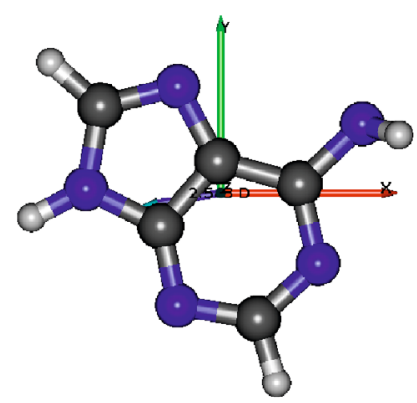

(e) $2 \mathrm{~b}$

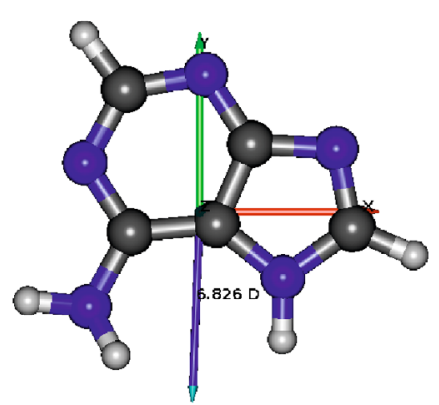

(c) $1 \mathrm{c}$

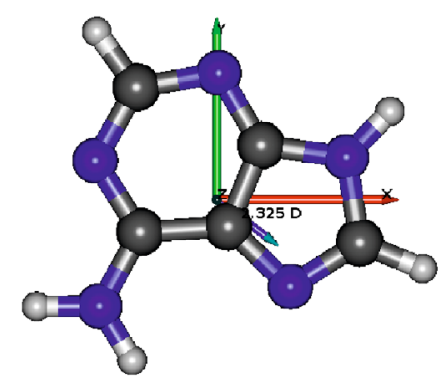

(f) $2 \mathrm{c}$

Fig. 4. Molecular geometries of various tautomers of adenine. The blue arrow shows the molecular dipole moment in the $x y$ plane.

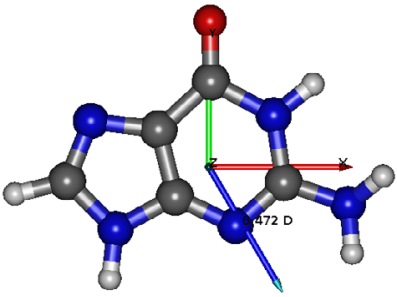

(a) 1

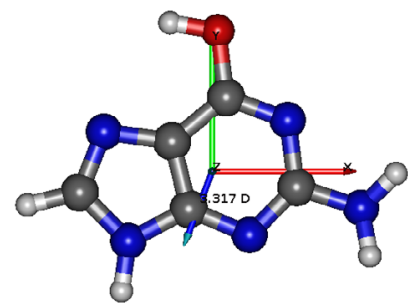

(d) 4

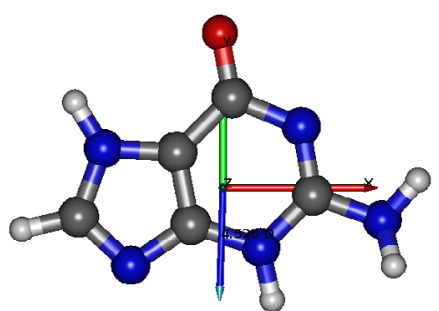

(g) 6

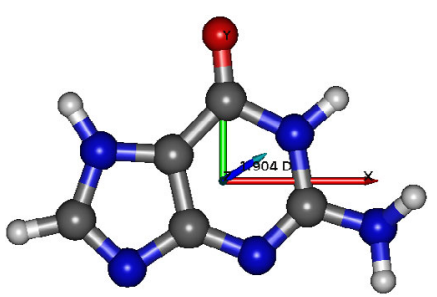

(b) 2

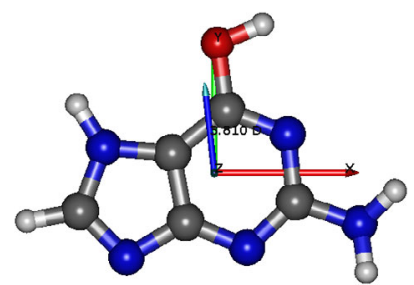

(e) $5 \mathrm{a}$

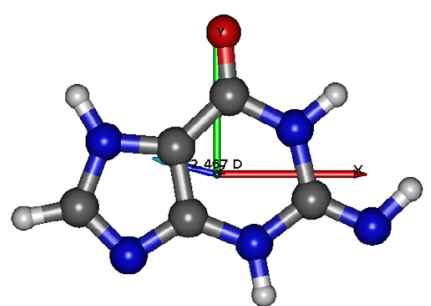

(h) 7

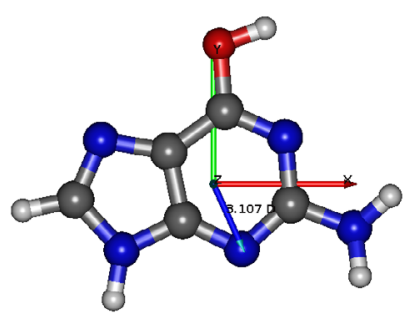

(c) 3

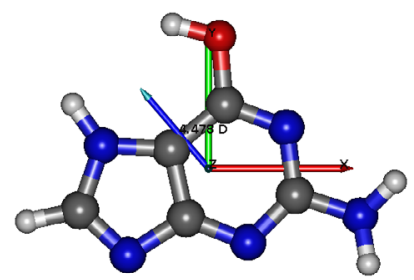

(f) $5 \mathrm{~b}$

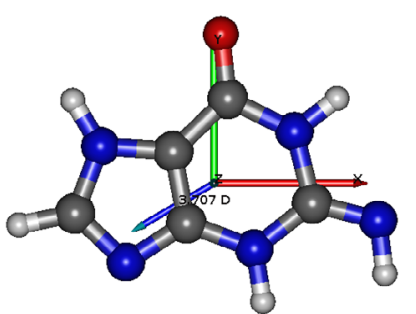

(i) 8

Fig. 5. Molecular geometries of various tautomers of guanine. The blue arrow shows the molecular dipole moment in the $x y$ plane. 
Table 2. Molecular parameters of cytosine tautomers. Dipole moments are given in Debye), rotational constants $A, B$ and $C$ in $\mathrm{GHz}$, rotational energies $\varepsilon_{J \tau}$ in $10^{-6} \mathrm{eV}$ and relative energies in $\mathrm{kJ} / \mathrm{mol}$. See main text for more details.

\begin{tabular}{cccccc}
\hline Tautomer & 1 & $2 \mathrm{a}$ & $2 \mathrm{~b}$ & $3 \mathrm{a}$ & $3 \mathrm{~b}$ \\
\hline$\mu_{x}$ & 4.16 & 4.29 & 2.87 & 1.76 & 3.00 \\
$\mu_{y}$ & 4.50 & -1.30 & 1.02 & -4.03 & -6.19 \\
$\mu_{z}$ & 0.46 & 0.59 & 0.56 & 0.00 & 0.00 \\
$|\mu|$ & 6.15 & 4.52 & 3.10 & 4.40 & 6.88 \\
$A$ & 3.8421 & 3.8609 & 3.9302 & 3.8116 & 3.8441 \\
$B$ & 2.0074 & 2.0136 & 1.9939 & 1.9740 & 1.9582 \\
$C$ & 1.3189 & 1.3241 & 1.3234 & 1.3093 & 1.3063 \\
$\varepsilon_{1-1}$ & 24.192 & 24.295 & 24.501 & 23.928 & 23.996 \\
$\varepsilon_{10}$ & 21.345 & 21.444 & 21.728 & 21.178 & 21.300 \\
$\varepsilon_{11}$ & 13.757 & 13.803 & 13.720 & 13.579 & 13.501 \\
$\Delta E$ & 0.0 & 9.357 & 6.189 & 54.812 & 71.584 \\
\hline
\end{tabular}

Table 3. Molecular parameters of adenine tautomers. Dipole moments are given in Debye), rotational constants $A, B$ and $C$ in $\mathrm{GHz}$, rotational energies $\varepsilon_{J \tau}$ in $10^{-6} \mathrm{eV}$ and relative energies in $\mathrm{kJ} / \mathrm{mol}$. See main text for more details.

\begin{tabular}{ccccccc}
\hline Tautomer & $1 \mathrm{a}$ & $1 \mathrm{~b}$ & $1 \mathrm{c}$ & $2 \mathrm{a}$ & $2 \mathrm{~b}$ & $2 \mathrm{c}$ \\
\hline$\mu_{x}$ & 0.36 & 2.10 & -0.27 & -4.29 & -2.51 & 1.86 \\
$\mu_{y}$ & 6.35 & 4.63 & -6.79 & 1.37 & -0.41 & -1.39 \\
$\mu_{z}$ & 0.00 & 0.00 & 0.67 & 0.00 & 0.00 & -0.03 \\
$|\mu|$ & 6.36 & 5.08 & 6.83 & 4.50 & 2.54 & 2.33 \\
$A$ & 2.3268 & 2.3374 & 2.3811 & 2.3073 & 2.3327 & 2.3609 \\
$B$ & 1.5443 & 1.5471 & 1.5317 & 1.5657 & 1.5458 & 1.5624 \\
$C$ & 0.9329 & 0.9355 & 0.9330 & 0.9373 & 0.9342 & 0.9402 \\
$\varepsilon_{1-1}$ & 16.010 & 16.065 & 16.182 & 16.018 & 16.040 & 16.225 \\
$\varepsilon_{10}$ & 13.481 & 13.536 & 13.706 & 13.419 & 13.511 & 13.652 \\
$\varepsilon_{11}$ & 10.245 & 10.267 & 10.193 & 10.352 & 10.257 & 10.350 \\
$\Delta E$ & 83.856 & 60.884 & 32.076 & 64.736 & 63.264 & 0.0 \\
\hline & & & & & &
\end{tabular}

Table 4. Molecular parameters of guanine tautomers. Dipole moments are given in Debye), rotational constants $A, B$ and $C$ in $\mathrm{GHz}$, rotational energies $\varepsilon_{J \tau}$ in $10^{-6} \mathrm{eV}$ and relative energies in $\mathrm{kJ} / \mathrm{mol}$. See main text for more details.

\begin{tabular}{cccccccccc}
\hline Tautomer & 1 & 2 & 3 & 4 & $5 \mathrm{a}$ & $5 \mathrm{~b}$ & 6 & 7 & 8 \\
\hline$\mu_{x}$ & 3.26 & 1.46 & 1.26 & -1.16 & -0.40 & -2.88 & -0.14 & -2.39 & -3.20 \\
$\mu_{y}$ & -5.54 & 0.92 & -2.76 & -3.04 & 3.73 & 3.38 & -4.47 & 0.60 & -1.88 \\
$\mu_{z}$ & 0.76 & 0.82 & 0.68 & 0.64 & 0.67 & 0.61 & 0.70 & -0.00 & 0.00 \\
$|\mu|$ & 6.47 & 1.90 & 3.11 & 3.32 & 3.81 & 4.48 & 4.53 & 2.47 & 3.71 \\
$A$ & 1.8995 & 1.8967 & 1.9001 & 1.9089 & 1.8870 & 1.8735 & 1.9294 & 1.8943 & 1.9108 \\
$B$ & 1.1102 & 1.1166 & 1.1251 & 1.1293 & 1.1282 & 1.1284 & 1.1063 & 1.1167 & 1.1113 \\
$C$ & 0.7011 & 0.7034 & 0.7069 & 0.7097 & 0.7063 & 0.7045 & 0.7037 & 0.7026 & 0.7026 \\
$\varepsilon_{1-1}$ & 12.447 & 12.462 & 12.511 & 12.565 & 12.470 & 12.415 & 12.555 & 12.453 & 12.498 \\
$\varepsilon_{10}$ & 10.755 & 10.753 & 10.782 & 10.830 & 10.725 & 10.662 & 10.890 & 10.740 & 10.808 \\
$\varepsilon_{11}$ & 7.491 & 7.527 & 7.577 & 7.606 & 7.587 & 7.580 & 7.486 & 7.524 & 7.502 \\
$\Delta E$ & 3.031 & 0.0 & 9.368 & 10.728 & 20.884 & 51.224 & 27.295 & 23.316 & 24.763 \\
\hline
\end{tabular}

to be rather substantial, as expected from multielectron systems as the nucleobases. The interaction between the positron and the permanent dipoles of the molecular partners is the one with the largest range of action $\left(1 / r^{2}\right)$ and with a marked angular dependence guided by the $l=1$ Legendre Polynomial. Hence, there will be well separated trajectories, classically speaking, which will undergo attractive interaction and others which will instead undergo largely repulsive interactions. The net result is therefore expected to be that of generating fairly large cross sections at low collision energies. The molecular geometries of the equilibrium structures are reported by Figures 1 and 2, the Oxygen and Nitrogen atoms corresponding to the negatively charged regions of each molecular environment, as we shall further discuss below. A global presentation of all computed integral cross sections (ICSs) is given in Figure 6, where the elastic (rotationally summed) values are given between about $1.0 \mathrm{eV}$ and $25 \mathrm{eV}$ of collision energy. For comparison we have also included the ICS for positron scattering from uracil, which is computed with a similar computational procefure [19]. The corresponding dipole moment values for each molecule are given in the figure. The numerical convergence of the values of the ICSs with respect to the number of contributing partial waves within the close-coupling (CC) part of the calculations is converged within a few percent for the maximum value 


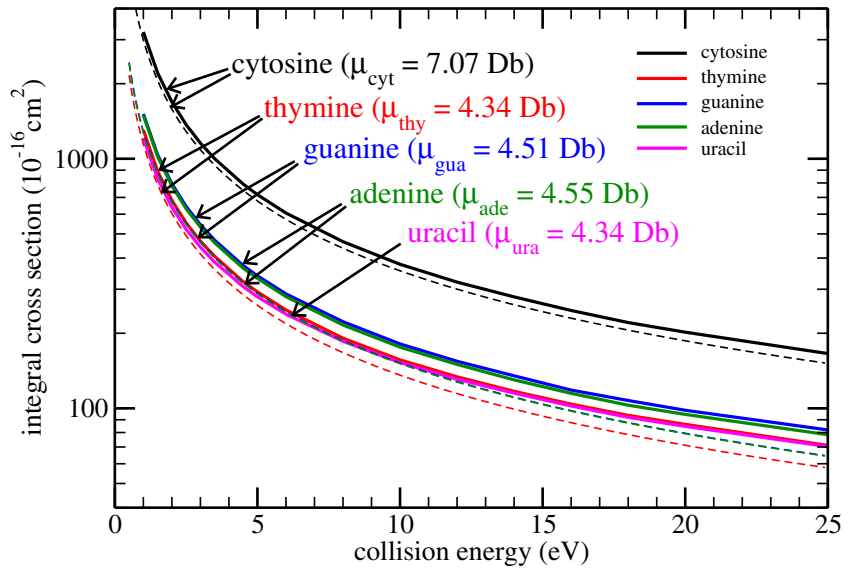

Fig. 6. Computed integral cross sections for positron scattering off the molecules are confined to the point group $C_{\mathrm{s}}$ and shown in Figures 1 and 2: cytosine (black lines), thymine (red lines), guanine (blue lines), adenine (green lines). In addition results for uracil (magenta line), as computed by Franz and Gianturco [19], are shown. The thick solid lines show he close coupling results and include the summation over rotational elastic and inelastic channels and include the Born-dipole correction for the $J=0$ to the $J^{\prime}=1$ transitions. The thinner, dashed lines show the Born-dipole results and include only the $J=0$ to $J^{\prime}=1$ transitions.

of $L=40$. For comparison also the Born-dipole results are shown (see dashed lines). One can further draw the following features from the data presented in Figure 6:

1. The cross sections scale roughly with the square of the dipole moment, as can be expected from equation (A.7). At the highest energies considered, i.e. from 20 to $25 \mathrm{eV}$, they are just below $100 \AA^{2}$ for guanine, adenine and thymine, while for cytosine (with the largest dipole) they reach nearly $200 \AA^{2}$. At $2.0 \mathrm{eV}$ the ICS for thymine $(|\mu|=4.34 \mathrm{Db})$ is around $750 \AA^{2}$, while at the same energy the ICS value for cytosine $(|\mu|=7.07 \mathrm{Db})$ is about $1800 \AA^{2}$.

2. Over the range of energies studied here, i.e. from 1 to $25 \mathrm{eV}$, all cross sections show a qualitatively similar behaviour w.r.t. the collision energy.

3. The cross sections do not show any features, no resonance structures can be seen.

4. The Born-dipole approximation is in good agreement with the more elaborate close-coupling calculations.

The data of Figure 7 report another interesting feature of positron scattering in the gas phase, i.e. the behaviour of the momentum-transfer cross sections (MTCSs). Such quantitites, in fact, measure the amount of deflection that the impinging particles experience from the nucleobases, hence provide, albeit only in the gas-phase in the present study, important indicators of the stength of the stopping power occurring for positrons before the onset of the other processes that can cause annihilation, positronium formation and molecular ionization. The larger the momentum-transfer cross sections the stronger the positron interactions with the target considered. Figure 7 shows the close-coupling results (solid lines) together

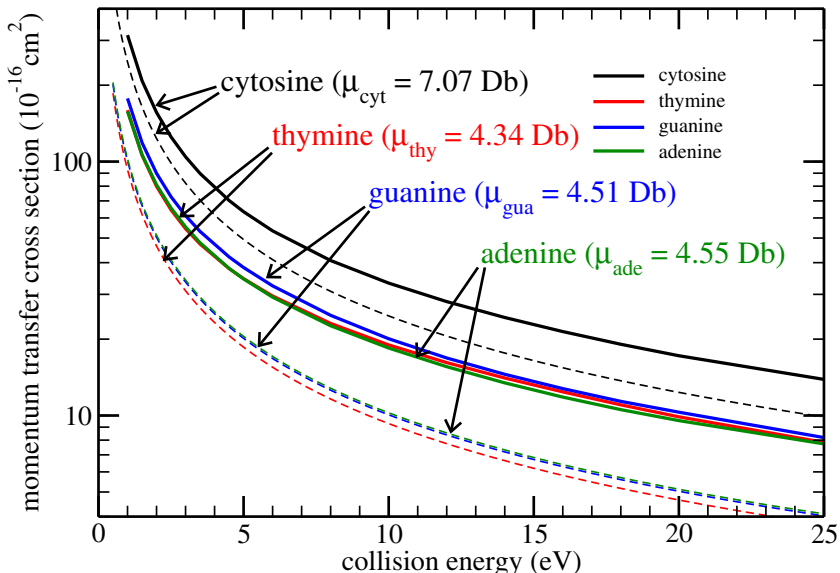

Fig. 7. Computed momentum cross sections for positron scattering off the molecules are confined to the point group $C_{\mathrm{s}}$ and shown in Figures 1 and 2: cytosine (black lines), thymine (red lines), guanine (blue lines), adenine (green lines). The thick solid lines show he close coupling results and include the summation over rotational elastic and inelastic channels and include the Born-dipole correction for the $J=0$ to the $J^{\prime}=1$ transitions. The thinner, dashed lines show the Born-dipole results and include only the $J=0$ to $J^{\prime}=1$ transitions.

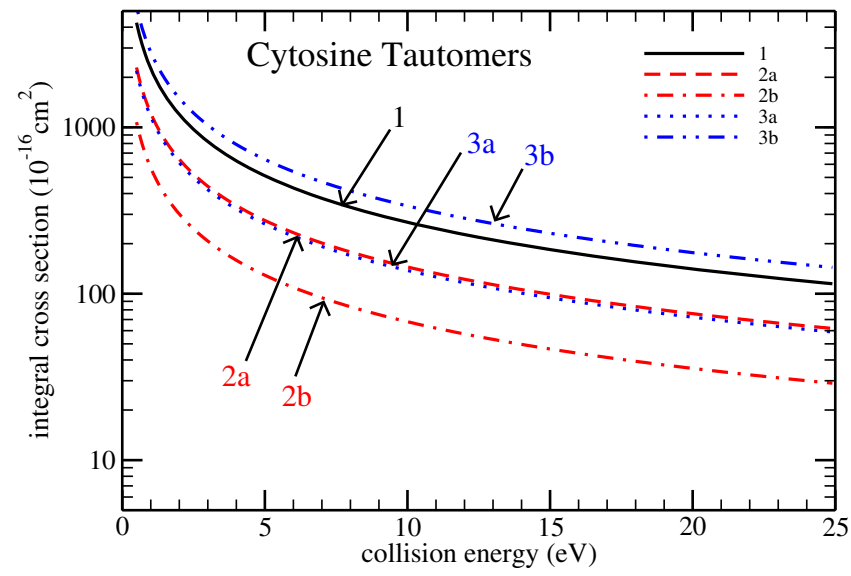

Fig. 8. Computed integral cross sections for positron scattering off various tautomers of cytosine. See text for details.

with the Born-dipole results. The Born MTCSs reproduce the qualitative behaviour of the close-coupling MTCSs, however, the former are roughly $50 \%$ lower than the latter.

Because of the good qualitative and quantitative agreement for the ICSs and the good quantitative agreement for the MTCSs between the Born-corrected close-coupling results and the pure Born-results, we performed Borncalculations for all tautomers shown in Figures 3-5. The ICSs and MTCSs for the tautomers of cytosine are given in Figures 8 and 9. One clearly sees that the overall qualitative behaviour of all cross sections are the same. Due to the differences in the magnitude of the molecular dipole moments (between 3.10 Debey for tautomer 2b and 6.88 Debey for tautomer $3 \mathrm{~b}$ ), the cross sections differ considerably in size. The situation is similar for tautomers of adenine, for which the ICSs and MTCSs are given in 


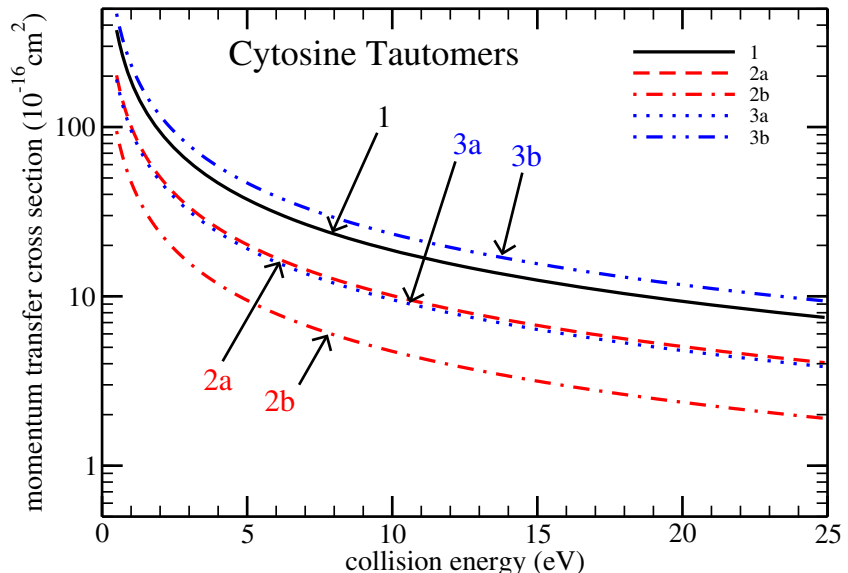

Fig. 9. Computed integral cross sections for positron scattering off various tautomers of cytosine. See text for details.

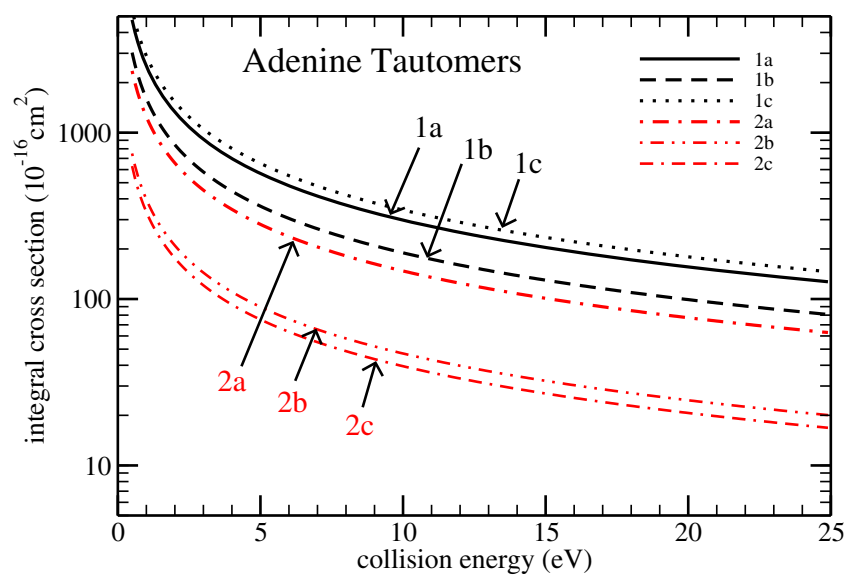

Fig. 10. Computed integral cross sections for positron scattering off various tautomers of adenine. See text for details.

Figures 10 and 11, respectively: all cross section show the same qualitative behaviour but vary considerable in their magnitude, due to the differences in the molecular dipole moment (between 2.33 Debey for tautomer 2c and 6.83 Debey for tautomer 1c). For the guanine tautomers shown in Figure 5 the ICSs are given in Figure 12 and the MTCSs in Figure 13. The corresponding dipole moments are between 1.90 Debey for tautomer 2 and 6.47 Debey for tautomer 1 (see Sect. 4).

\section{Present conclusions}

In order to more clearly summarize our present findings and consider collectively their significance in terms of their molecular properties as listed by Table 1, a comparison of the final values of the ICS and MTCS, corrected by the Born terms for the higher partial waves and added of the inelastic contributions from rotational excitation processes that are opened at the energies considered, is reported by the data of Figures 6 and 7, respectively. We clearly see there that the molecules with the similar dipoles (from thymine to adenine) also exhibit fairly similar sizes for their ICS values provided by our calculations. On the other hand, the cytosine cross sections are

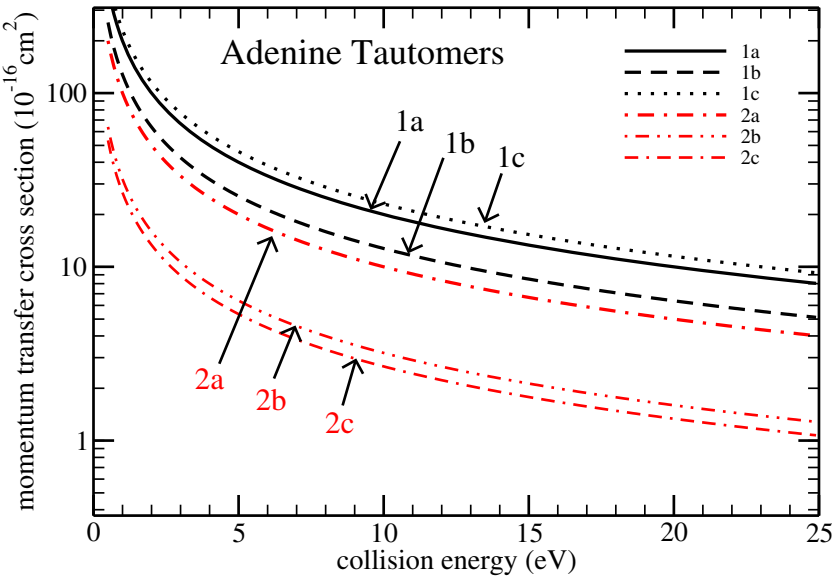

Fig. 11. Computed integral cross sections for positron scattering off various tautomers of adenine. See text for details.

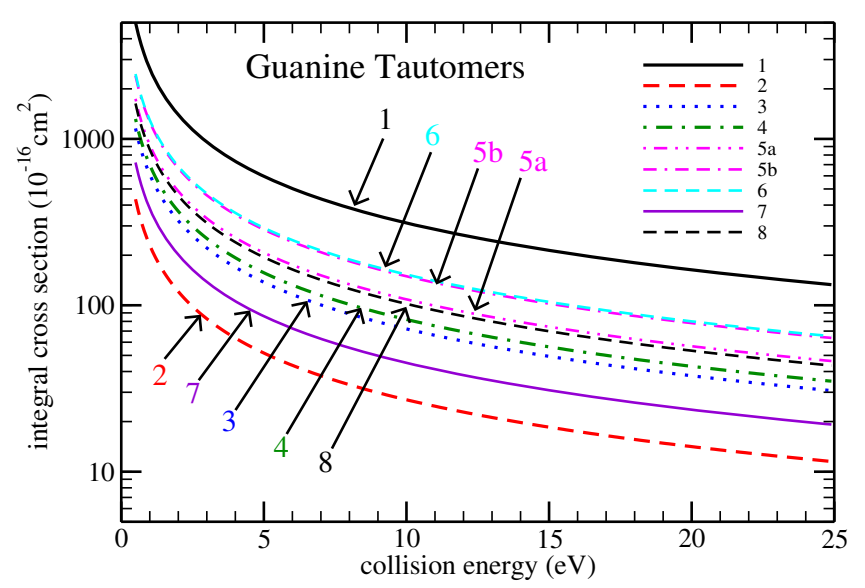

Fig. 12. Computed integral cross sections for positron scattering off various tautomers of guanine. See text for details.

much larger at all energies, and dramatically so at those collision energies where the dipole moments dominate the positron-molecule interactions.

One should also note here that in the present calculations we have been considering solely the elastic scattering components of the total cross sections for the molecules studied here, adding only the still important rotational excitation channels but disregarding for the moment the effects from other significant channels associated with Ps formation and positron annihilation processes. Such an approach is partly justified by noting that most experimental studies also limit themselves to measuring the elastic channels without resolving rotational and vibrational inelastic components, thereby making a comparison with our calculations also easier to carry out. On the other hand, we are well aware of the fact that such "reactive" channels acquire great significance after their respective channel openings [1] and therefore we plan to further consider in our future work the possible modelings of such effects within our scattering calculations.

Currently there is no experimental data from gas-phase scattering experiments available, with which we could compare our computed results. Certainly, in the gas phase 


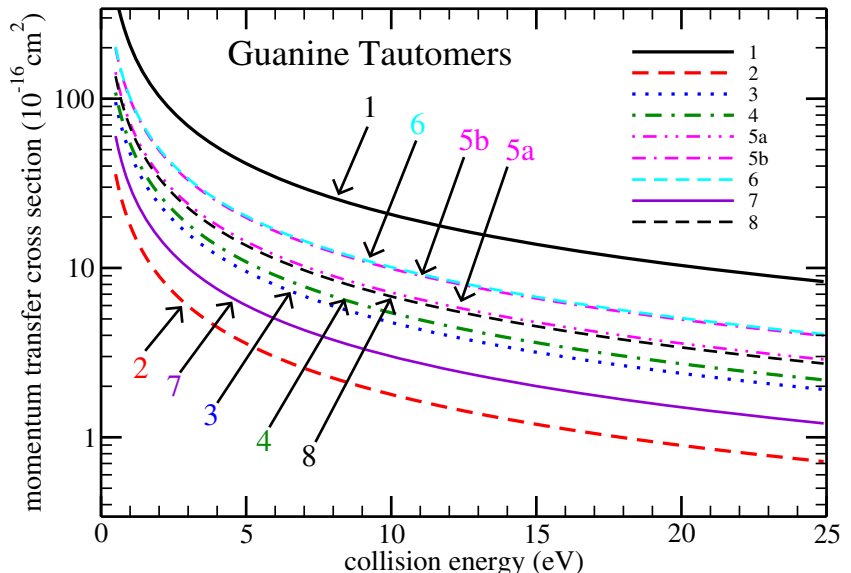

Fig. 13. Computed integral cross sections for positron scattering off various tautomers of guanine. See text for details.

experiments on will always work with a mixture of various tautomers, so that the measured cross sections will be superpositions of cross sections from the individual molecular species. Several groups of experimentalists around the globe are working on setting up such epxeriments. All these experiments are using a linear transmission technique. This technique has the disadvantage that scattering events with a scattering angle smaller than a certain angle $\theta_{\text {min }}$ cannot be distinguished from the unscattered particles of the positron beam and therefore all scattering events within this angular cone in forward direction are missing in the cross sections (see e.g. Sullivan et al. [20] for a general discussion). In order to compare experimental and computational integrated cross sections, one therefore needs to add the estimated partially integrated differential cross section, which includes all scattering events into forward direction with scattering angles smaller than the angular discrimination angle $\theta_{\text {min }}$. In our recent work on positron scattering off pyrimidine [9] and tetrahydrofuran [21] we have compared computed cross sections with experimental data in this way.

This can be done by an integration over the computed differential cross sections $\frac{d \sigma_{\text {calc }}}{d \Omega}$ carried out between the forward direction and the angular discrimination angle $\theta_{\min }$

$$
\Delta \sigma^{\text {forward }}=2 \pi \int_{0}^{\theta_{\min }} \frac{d \sigma_{\text {calc }}}{d \Omega} \sin \theta d \theta .
$$

For the molecular targets considered here, reasonable results can be obtained with the Born dipole approximation. The differential cross section within the Born dipole approximation is given in equation (A.4) in the appendix. The above integral can be solved analytically and one obtains

$$
\Delta \sigma_{01}^{\mathrm{T}}=\frac{4 \pi}{3 k_{00}^{2}} \sum_{\tau^{\prime}} D_{\tau^{\prime}}^{2} \ln \frac{\left(k_{00}+k_{1 \tau^{\prime}}^{\prime}\right)^{2}}{k_{00}^{2}+k_{1 \tau^{\prime}}^{\prime 2}-2 k_{00} k_{1 \tau^{\prime}}^{\prime} \cos \theta_{\min }} .
$$

All symbols are explained in the appendix. All quantities needed to compute $\Delta \sigma_{01}^{\mathrm{T}}$ are given in the Tables. Some of the currently employed scattering machines are using a retarding potential technique. Kauppila et al. [22] and Kwan et al. [23] are giving an equation to estimate the angle discrimination angle $\theta_{\text {min }}$ as a function of the retarding potential $\Delta V$ and the collision energy $E$ by the following relation

$$
\theta_{\min }=\sin ^{-1} \sqrt{\frac{e \Delta V}{E}} .
$$

Here $e$ is the elementary charge. The physical idea behind this equation is as follows: After the collision the positrons velocity has a component in axial direction and a component in transversal direction. The corresponding contributions of the kinetic energy are $E_{\text {axial }}=E \cos ^{2} \theta$ and $E_{\text {trans }}=E \sin ^{2} \theta$. The retarding potential is located between the scattering cell and the detector. The potential affects only the axial part of the kinetic energy. All scattered particles with a transverse kinetic energy, that is larger than the retarding potential are reaching the detector, and, hence, are counted as unscattered particles. The minimal transversal kinetic energy for this to happen is

$$
E_{\text {trans }}=e \Delta V
$$

which gives the expression above for the angular discrimination error $\theta_{\min }$.

This work has been supported by the COST Action MP $1002-$ Nano-scale Insights into Ion Beam Cancer Therapy. The financial support of CINECA computational grants is gratefully acknowledged. We thank Isabella Baccarelli and Nico Sanna of CINECA for their initial advices with the SCELib software package. We thank A.-R. Allouche for making the program Gabedit [24] freely available, with which Figures 1-5 are generated.

\section{Appendix A: Expressions for the first Born approximation using the asymptotic part of the dipole interaction}

In this Appendix we present general equations for the first Born approximation for polar molecules with low molecular symmetry, e.g. point groups $C_{\mathrm{s}}$ and $C_{1}$. Equations are given for the $\mathbf{K}$-matrix elements, the total cross section and the momentum transfer cross section. All equations are using the asymptotic part of the dipole interaction. We have implemented all equations into a local version of the program POLYDCS. The original implementation in POLYDCS presented by Sanna and Gianturco [8] only deals with molecules, in which the molecular dipole moment is either zero or is aligned along one of the principal axis of inertia, which is also an axis of symmetry.

\section{A.1 K-matrix elements}

In the first Born approximation the elements of the $\mathbf{K}$-matrix are given by [4]

$$
\begin{aligned}
\mathbf{K}_{l h, l^{\prime} h^{\prime}}^{p \mu}= & -2 k \int_{0}^{\infty} j_{l}(k r) j_{l^{\prime}}(k r) r^{2} d r\left\langle\mathbf{X}_{l h}^{p \mu}\right| \\
& \times \sum_{\lambda H} v_{\lambda H}(\mathbf{r}) \mathbf{X}_{\lambda H}^{A}\left|\mathbf{X}_{l^{\prime} h^{\prime}}^{p \mu}\right\rangle .
\end{aligned}
$$


For low-symmetry molecules the potential can include dipole terms in the direction of each coordinate axis.

$$
\sum_{\lambda H} v_{\lambda H}(\mathbf{r}) \mathbf{X}_{\lambda H}^{A}=\frac{A_{-1}}{r^{2}} S_{1}^{-11}+\frac{A_{0}}{r^{2}} S_{1}^{01}+\frac{A_{1}}{r^{2}} S_{1}^{11} .
$$

Here the functions $S_{l}^{m q}$ are real spherical harmonics, as defined in Gianturco and Jain [4]. The coefficients $A_{m}$ are related to the components of the dipole moment

$$
A_{m}=-\left(\frac{4 \pi}{3}\right)^{1 / 2} D_{m}
$$

\section{A.2 Differential cross section}

The generalized form of the differential cross section is given by

$$
\frac{d \sigma_{01}^{\mathrm{T}}}{d \Omega}=\sum_{\tau^{\prime}} \frac{4 D_{\tau^{\prime}}^{2}}{3} \frac{k_{1 \tau^{\prime}}}{k_{00}} \frac{1}{\left(k_{00}^{2}+k_{1 \tau^{\prime}}^{2}-2 k_{00} k_{1 \tau^{\prime}} \cos \theta\right)},
$$

where $D_{\tau^{\prime}}$ is the component of the molecular dipole moment along the principal axis of inertia associated with the quantum number $\tau^{\prime} . \theta$ is the angle between the positron momenta before and after the collision. The two momenta are given by

$$
k_{00}=\sqrt{2 E_{\mathrm{scat}}} \quad \text { and } \quad k_{1 \tau^{\prime}}^{\prime}=\sqrt{2\left(E_{\mathrm{scat}}-\omega_{\tau^{\prime}}\right)},
$$

where

$$
\omega_{\tau^{\prime}}=\varepsilon_{1 \tau^{\prime}}-\varepsilon_{00}
$$

is the energy difference between the rotational ground state and the rotational level $J^{\prime}=1$ with sublevel $\tau^{\prime}$.

\section{A.3 Total integral cross section}

The generalized form of the total integral cross section is given by

$$
\sigma_{01}^{\mathrm{T}}=\frac{8 \pi}{3 k_{00}^{2}} \sum_{\tau^{\prime}} D_{\tau^{\prime}}^{2} \ln \left|\frac{k_{00}+k_{1 \tau^{\prime}}^{\prime}}{k_{00}-k_{1 \tau^{\prime}}^{\prime}}\right|
$$

\section{A.4 Momentum transfer cross section}

With the same definitions as above a generalized definition of the momentum transfer cross section is given by

$$
\sigma_{01}^{\mathrm{M}}=\frac{8 \pi}{3 k_{00}^{2}} \sum_{\tau^{\prime}} D_{\tau^{\prime}}^{2}\left(1-\frac{\left(k_{00}-k_{1 \tau^{\prime}}^{\prime}\right)^{2}}{2 k_{00} k_{1 \tau^{\prime}}^{\prime}} \ln \left|\frac{k_{00}+k_{1 \tau^{\prime}}^{\prime}}{k_{00}-k_{1 \tau^{\prime}}^{\prime}}\right|\right) .
$$

\section{References}

1. Radiation Damage in Biomolecular Systems, edited by G. Garcia Gómez-Tejedor, M.C. Fuss (Springer, Berlin, 2012)

2. B. Boudaiffa, P. Clouthier, D. Hunting, M.A. Huels, L. Sanche, Science 287, 1658 (2000)

3. New Directions in Antimatter Chemistry and Physics, edited by C.M. Surko, F.A. Gianturco (Kluwer Ac. Publ., Dordrecht, 2000)

4. F.A. Gianturco, A. Jain, Phys. Rep. 143, 347 (1986)

5. E.S. Chang, U. Fano, Phys. Rev. A 6, 173 (1972)

6. R. Curik, F.A. Gianturco, N. Sanna, J. Phys. B 33, 2705 (2000)

7. A. Jain, F.A. Gianturco, J. Phys. B 24, 2387 (1991)

8. N. Sanna, F.A. Gianturco, Comput. Phys. Commun. 114, $142(1998)$

9. J. Franz, F.A. Gianturco, Phys. Rev. A 88, 042711 (2013)

10. E. Boronski, R.M. Nieminen, Phys. Rev. 34, 3820 (1986)

11. J. Franz, F.A. Gianturco, K.L. Baluja, J. Tennyson, R. Carey, R. Montuoro, R.R. Lucchese, T. Stoecklin, P. Nicholas, T.L. Gibson, Nucl. Instrum. Methods B 266, 425 (2008)

12. J.P. Perdew, K. Burke, M. Ernzerhof, Phys. Rev. Lett. 77, 3865 (1996)

13. J.P. Perdew, K. Burke, M. Ernzerhof, Phys. Rev. Lett. 78, 1396 (1997)

14. M.J. Frisch et al., Gaussian 09, Revision B.01 (Gaussian, Inc., Wallingford CT, 2010)

15. A.L. Hickey, C.N. Rowley, J. Phys. Chem. A 118, 3678 (2014)

16. N. Sanna, I. Baccarelli, G. Morelli, Comput. Phys. Commun. 180, 2544 (2009)

17. N. Sanna, I. Baccarelli, G. Morelli, Comput. Phys. Commun. 180, 2550 (2009)

18. A. Jain, D.G. Thompson, Comput. Phys. Commun. 32, 367 (1984)

19. J. Franz, F.A. Gianturco, I. Baccarelli, Eur. J. Phys. D 68, 183 (2014)

20. J.P. Sullivan, C. Makochekanwa, A. Jones, P. Caradonna, D.S. Slaughter, J. Machacek, R.P. McEachran, D.W. Mueller, S.J. Buckman, J. Phys. B 44, 035201 (2011)

21. J. Franz, F.A. Gianturco, J. Chem. Phys. 139, 204309 (2013)

22. W.E. Kauppila, T.S. Stein, J.H. Smart, M.S. Dababneh, Y.K. Ho, J.P. Downing, V. Pol, Phys. Rev. A 24, 725 (1981)

23. C.K. Kwan, W.E. Kauppila, R.A. Lukaszew, S.P. Parikh, T.S. Stein, Y.J. Wan, M.S. Dababneh, Phys. Rev. A 44, 1620 (1991)

24. A.-R. Allouche, J. Comput. Chem. 32, 174 (2011)

Open Access This is an open access article distributed under the terms of the Creative Commons Attribution License (http://creativecommons.org/licenses/by/4.0), which permits unrestricted use, distribution, and reproduction in any medium, provided the original work is properly cited. 